\title{
Consent for mobile phone surveys of non-communicable disease risk factors in low-resource settings: an exploratory qualitative study in Uganda
}

\author{
Erisa Mwaka ${ }^{1}$, Janet Nakigudde ${ }^{1}$, Joseph $\mathrm{Ali}^{2,3}$, Joseph Ochieng ${ }^{1}$, Kristina Hallez ${ }^{3}$, Raymond Tweheyo ${ }^{1}$, \\ Alain Labrique $^{2}$, Dustin G. Gibson ${ }^{2}$, Elizeus Rutebemberwa ${ }^{1}$, George Pariyo ${ }^{2}$ \\ ${ }^{1}$ Makerere University of College Health Sciences, Kampala, Uganda; ${ }^{2}$ Johns Hopkins Bloomberg School of Public Health, Baltimore, USA; ${ }^{3}$ Johns \\ Hopkins Berman Institute of Bioethics, Baltimore, USA \\ Contributions: (I) Conception and design: J Ali, E Mwaka, G Pariyo, A Labrique, DG Gibson, E Rutebemberwa; (II) Administrative support: R \\ Tweheyo, K Hallez, E Rutebemberwa; (III) Provision of study materials or patients: J Nakigudde, J Ali, E Mwaka, K Hallez, R Tweheyo, G Pariyo, \\ DG Gibson; (IV) Collection and assembly of data: E Mwaka, J Nakigudde, J Ali, J Ochieng; (V) Data analysis and interpretation: All authors; (VI) \\ Manuscript writing: All authors; (VII) Final approval of manuscript: All authors. \\ Correspondence to: Erisa Mwaka. Makerere University College of Health Sciences, P. O. Box 7072, Kampala, Uganda. Email: erisamwaka@gmail.com.
}

Background: Lack of data for timely decision-making around the prevention and control of non-
communicable diseases (NCDs) presents special challenges for policy makers, especially in resource-limited
settings. New data collection methods, including pre-recorded Interactive Voice Response (IVR) phone
surveys, are being developed to support rapid compilation of population-level disease risk factor information
in such settings. We aimed to identify information that could be used to optimize consent approaches for
future mobile phone surveys (MPS) employed in Uganda and, possibly, similar contexts. Methods: We conducted an in-depth qualitative study with key stakeholders in Uganda about consent approaches, and potential challenges, for pre-recorded IVR NCD risk factor surveys. Semi-structured interviews were conducted with 14 key informants. A contextualized thematic approach was used to interpret the results supported by representative quotes.

Results: Several potential challenges in designing consent approaches for MPS were identified, including low literacy and the lack of appropriate ways of assessing comprehension and documenting consent. Communication with potential respondents prior to the MPS and providing options for callbacks were suggested as possible strategies for improving comprehension within the consent process. "Opt-in" forms of authorization were preferred over "opt-out". There was particular concern about data security and confidentiality and how matters relating to this would be communicated to MPS respondents.

Conclusions: These local insights provide important information to support optimization of consent for MPS, whose use is increasing globally to advance public health surveillance and research in constructive ways.

Keywords: Mobile phone surveys (MPS); consent; mHealth; public health surveillance; Uganda

Received: 10 April 2019; Accepted: 19 July 2019; Published: 19 August 2019.

doi: 10.21037/mhealth.2019.07.05

View this article at: http://dx.doi.org/10.21037/mhealth.2019.07.05

\section{Introduction}

Lack of data for timely decision-making presents special challenges for policy makers with regard to prevention and control of non-communicable diseases (NCD), especially in resource limited settings (1). In response to the insufficient data on NCD surveillance, the World Health Organization (WHO) called for innovative surveillance systems to strengthen the monitoring of NCDs and associated risk 
factors (2). This call was echoed in 2015 at a United Nations meeting that adopted 17 Sustainable Development Goals (SDGs); of which, the third goal calls for the reduction in premature mortality from NCDs through prevention, treatment and promotion of mental health and well-being (3). One such innovative approach to increase data availability is the use of mobile and digital health technologies to support disease surveillance (4). To mitigate cost and time requirements associated with household data collection for public health surveillance, several highincome countries (HICs) have devised and employed digital health approaches to collect population-wide health and demographic information $(5,6)$; however, such approaches are only beginning to be introduced in many low- and middle-income countries (LMICs) (7).

The global proliferation of mobile phones has, in particular, impacted public health surveys, especially in LMICs where hitherto face-to-face household interviews have been used (8). Face-to-face interviews can be expensive to implement, require a considerable amount of training and time, and are often difficult to conduct in hard to reach or conflict regions $(9,10)$. Leveraging the high coverage rates of mobile phone use in many LMICs, it is now possible to remotely conduct interviews-either though a live interviewer or pre-recorded messages-on a variety of public health topics through the use of relatively short mobile phone surveys (MPS). In addition to being used for disease and risk factor surveillance, MPS could potentially play a role in monitoring population-level reach, acceptability and effectiveness of a variety of public health interventions in order to inform rapid program and policy evaluation (1). Much as MPS have numerous advantages, some limitations remain. The utility of MPS at a population-level is likely associated with mobile phone distribution across sub-populations, with young, urban populations being more likely to have a mobile phone compared to the rural individuals; battery or connectivity/ network failure that leads to dropped calls (11); multiple and communal phone ownership (8); and various ethics and regulatory challenges that may arise at the national, community or individual levels (12).

Moreover, the nature of challenges to successful implementation of MPS may vary across countries or communities, generating the need for independent consultations from experts and other stakeholders wherever MPS is being conducted (12). Continuous local stakeholder engagement and collaborative partnership is imperative for the effective use of MPS. On-going stakeholder engagement can contribute to positive attitudes towards MPS and the eventual acceptability of this approach as a routine method of collecting data for public health use (12).

From an ethics and regulatory perspective, understanding norms and requirements associated with consent for participation in MPS is of particular interest, given desires to incorporate concise consent disclosures and authorizations into mobile surveys, while aligning consent practices with local cultural and regulatory expectations. The notion of informed consent has evolved significantly over the last century, first in medical care, then in medical research (13) and now in digital health contexts. Discussions have emerged about how best to design and execute informed consent processes using portable electronic devices and mobile phones $(12,14,15)$ particularly for population-wide studies. In-roads have been made in designing digital informed consent models for clinical care and research (16) but little is documented regarding consent for MPS designed to inform public health policies and practices in LMICs.

As part of the Bloomberg Philanthropies Data for Health Initiative (D4H) (17-19), we conducted an exploratory qualitative study to capture the views of key stakeholders in Uganda in order to better understand how best to conduct consent for pre-recorded Interactive Voice Response (IVR) NCD risk factor surveys, and what consent-related challenges are likely to arise. Our goal was to identify information that could be used to optimize consent approaches for future MPS employed in Uganda, and learn lessons that might be applied in similar contexts elsewhere.

\section{Methods}

This was a cross-sectional study that employed key informant (KI) interviews to explore disclosure language and authorization approaches for IVR-based NCD surveys to optimize MPS consent approaches. The study population comprised 14 purposively selected KIs, that included officials or experts with knowledge of NCD and/ or related mHealth survey approaches, officials responsible for oversight of health research, bioethics scholars, research ethics committee (RECs) members, and MPS technical experts in Uganda. At the beginning of each KI interview, a hypothetical scenario (based on planned activities) of an IVR for NCD risk factor MPS was read to the respondent to help contextualize the discussion. Using an interview guide developed by study team members, the semistructured interviews explored KIs' MPS experience and 
Table 1 Demographic characteristic

\begin{tabular}{lc}
\hline Characteristic & $\begin{array}{c}\text { Frequency } \\
(\mathrm{n}=14)\end{array}$ \\
\hline Gender & 11 \\
Male & 3 \\
Female & \\
Education & 2 \\
Bachelors & 7 \\
Masters & 5 \\
PhD & \\
Occupation & 6 \\
Oversight of health research/ethicist & 3 \\
MPS technical expert & 5 \\
Researcher/investigator & 10 \\
Place of work & 2 \\
University & \\
National research regulatory body (Uganda & \\
National Council for Science and Technology) & \\
Non-governmental organization & \\
Hospital & \\
\hline
\end{tabular}

ethics background; general impressions of the consent process; disclosure information during consent; modes of authorization; and other consent issues concerning the capacity and training of researchers and RECs with regard to MPS (the interview guide is available in Supplementary file 1). Interviews were conducted in the offices of the participants by two researchers and two research assistants with experience in qualitative research, and on average each KI lasted 1 hour.

All interviews were conducted in English, audio recorded and later transcribed verbatim. Debriefing meetings were held by the research team at the end of each interview to agree on the different perspectives that had arisen. Data analysis was done manually and began at the point of the data collection. The authors read the transcripts multiple times in order to familiarize themselves with the data. They then generated codes individually based on emergent themes and later agreed on these codes by consensus. The initial codes included general impressions about consent for MPS authorization language and other emerging ethical considerations. A contextualized thematic approach (20) was used to interpret the results supported by representative quotes.

\section{Ethical consideration}

All recordings and transcripts were de-identified, assigned special codes and stored on a password-protected computer. The study was approved by the Makerere University School of Public Health Higher Degrees and REC (Protocol version 445/10/2017) and the Johns Hopkins Bloomberg School of Public Health Institutional Review Board; and was conducted in accordance with the provisions in the Helsinki Declaration (21). Informed consent was obtained from all participants prior to interview.

\section{Results}

Fourteen KIs were interviewed; the majority were male (12/14) with a mean age of 42.4 years (range, $28-54$ years). All were residents of Uganda and were either affiliated to a university, research institution or Uganda National Council for Science and Technology. The participants' main job roles included research oversight/member of a REC, research/public health surveillance, and MPS technical experts (Table 1).

Nine had used a mobile phone for data collection and had also ever participated in a MPS. Almost all (13/14) had work-related obligations associated with NCDs or had ever conducted research on NCDs.

\section{Perceptions of the consent process for MPS}

KIs highlighted several potential ethical challenges and opportunities to conducting MPS for NCDs. When asked what some of the potential challenges are of obtaining consent for a MPS, some KIs wondered whether respondents of MPS adequately understand information presented during a "consent module". They argued that MPS tend to be brief and are not likely to be conducive to meeting the "understanding" requirement of informed consent in Uganda:

"Of course other general challenges would be in terms of, when you dial or call someone and they are listening, whether they are actually understanding and you give them adequate time to make a decision of whether they want to participate in the study or not." (KI 01)

One KI pointed out the lack of appropriate ways of assessing comprehension and the challenge of not having the 
benefit of non-verbal cues:

"The other problem I see is that when we do consenting on face to face, we look at the body language of the person and we can get an idea of who has understood what we are talking about and who hasn't. In the phone consent process we lose the body language information because we are talking across a distance somebody can tell you yes I understand and the body language is telling you something different." (KI 02)

One KI noted the need to identify appropriate ways of documenting informed consent for various types of MPS:

"For the text messages one can assume that the person can reply and say I accept, then for the voice how do you document that? Unless within this prerecorded touch pad there is a yes, No press 1 or press 2 to agree as a form of consent. But one has to think of a unique way of documenting the consent." (KI 08)

When asked what potential opportunities for consent were presented through mobile phones, respondents gave suggestions that could help improve comprehension of consent. Three KIs indicated that communication with potential respondents prior to the survey and providing options for callback could improve on understanding during the consent process:

"To give a person a telephone call and give the person some time to reflect and then call back and maybe ask the person to call back if they are interested that should be one of them. The other one is to send information by text in addition to voice so that you use both the voice and the text media." (KI 08)

\section{Public bealth surveillance versus research}

KIs were asked whether consent processes and requirements for MPS-based data collection for research differ from MPS data collection to support public health surveillance; and if so, in what ways. Respondents distinguished consent for activities that fell inside and outside the scope of routine public health surveillance. Several KIs suggested that the stringent consent disclosure language required for research would not necessarily apply to public health surveillance:

"I believe there should be different consent requirements. For example in the (research) consenting process we are interested in assessing the level of understanding; whether our participants have understood before consenting. Whereas for public health surveillance, and for public health services or for clinical care we do not require an assessment of [respondent] understanding." (KI 03)

"...Of course (the consent language ought to be) more detailed in research as compared to public health surveillance." (KI 01)

Public health surveillance was considered to be a routine activity involving the collection of data from the general public. It was perceived that potential participants had the right to decide whether to participate in research or not, but were generally obliged to participate in public health surveillance activities for the public good:

"The main difference is that in public health surveillance you are doing a service in which the respondent already knows that this is part of the normal life. If public health officials appeared, say at my house to find out whether I use mosquito nets or whether they were supplied I should take that as a normal thing, but to come in as research, I take this as something that is out of the norm, something that needs not to be done, something that is optional, something that may or may not happen...so the two are very different, requiring different disclosure language." (KI 03)

Concerning when MPS are to be used for research, some KIs believed that consent processes for MPS should follow standard international guidelines for consent and that the study information disclosed for MPS should be the same as in-person studies. For example, one respondent stated:

"There should be no reason to exclude anything just because you are using mobile health. In principle, I do not see why anything should be excluded because a mobile (phone survey) is just a convenient way of collecting data, it doesn't change anything! It is simply research." (KI 13)

Similarly, according to another respondent,

"...(T)he standard should be the same as a normal patient questionnaire, all the information that is presented for consent should be ethically presented, like the study purpose, the study funder, the data that is going to be collected and its use." (KI08)

\section{How much to disclose during the MPS consent process}

Some KIs discussed the need to establish a consent approach that provides adequate information, while also minimizing the survey time burden for participants. They opined that the MPS (and consent) should be of optimal duration so as not to inconvenience potential respondents:

"When a person gets a telephone call... usually these telephone calls find someone doing something including sleeping or resting and the respondents or the research participants did not plan to have this call. They did not expect (the call)... So what I think the bottom line is, people must be presented with adequate information. And the researcher has to find a way of delivering this adequate information within the shortest time possible." (KI 03)

Or, as stated by another respondent:

"The practical thing I see is making concise summaries that can be shared including through audio or, especially if you bave a 
smartphone option, you could actually write a text." (KI 04)

One KI defined adequate information as follows:

"A person should know what this (the mobile phone survey) is all about, be should know who is doing it, and (for what reason). The person should know the expected input in terms of time, effort, inconvenience and risk that may accrue from bis involvement." (KI 03)

One participant wondered whether norms for electronic disclosures in other digital contexts would be appropriate to adopt, and if so, whether disparities in types of mobile phone use could pose particular challenges:

"I know you also know that when you are logging onto something online they will give you the agreements. So I think the same principle should be used. Whether someone reads it or not that's another matter. So the challenge again is that if someone has a simple phone, as opposed to a smartphone, how are you going to relay all that (information) in a way that is not intrusive?" (KI 04)

\section{Options for bow MPS respondents might autborize their participation}

KIs were presented with examples of different approaches for MPS respondents to indicate their willingness to participate or not participate in a survey (opt-in vs. opt out; active $v s$. passive/implied agreement). Most KIs preferred active forms of authorization (e.g., "press 1 to continue" over passive forms (e.g., "by completing this survey you agree to participate"). One KI referred to the passive form as being a "lower level" form of consent. The KI also described a belief that the population was, in general, better at following positive directions than negative ones:

"Our people bere tend to understand the positive things rather that the negative ones. So if you say 'Press 1 if you like to participate' it's much better that 'Press 3 if you would not like to participate'; so our population bere tends to understand clearly more positive directions than the negative directions." (KI 03)

\section{Other ethics considerations}

Some KIs mentioned concerns about confidentiality for MPS research, noting that all mobile phone numbers are linked to national identity card numbers:

"(Even if random number dialing is used), a mobile phone number can be tracked back to the source. If someone lands on that information they would be in position to trace the identity of the participant. Remember that for our country, it is related to the national ID and other identifications. So that would be one of the challenges. That is something to think about." (KI 01)

They noted that participants might be worried about providing personal information given that it could be linked to their phone number:

"So in this situation the study says no identifying data will be collected but if I know that you have my telephone number you can trace who I am! So bow likely am I to give you consent to participate in your study, in which you are saying, you are not collecting identifying data? So the challenge is that some people know that you can identify them through these mobile phones and yet they may not want their identities to be revealed." (KI 04)

Another KI mentioned concerns around third parties, such as telecommunications companies and the police, receiving access to identifying information provided by MPS participants:

"Some of the challenges have included assurances of confidentiality because we know that mobile phone companies store the information, whether it is text or voice. And we know that this information can be retrieved. For example, it is usually retrieved for legal purposes and the police and security organs can be looking for information around particular calls for particular information...How do you assure a buman subject that information will be beld in confidence because once the information is out there whether in text or voice, it's not in the control of the investigator?...That has been the main problem and the investigators have not been able to conclusively ensure that confidentiality." (KI 03)

In summary, respondents highlighted several key issues pertaining to consent for MPS, which need to be better understood. First, is the issue of content of the consent, including a need to balance the need for MPS to remain relatively brief with the need to provide all key information. Second, documentation of consent; making sure that when an individual responds to a MPS he/she has intentionally and voluntarily given permission. Third, understanding of consent; ensuring that the respondent has a meaningful opportunity to understand the consent information, before making a decision about participation. Fourth, mode of authorization; respondents expressed preferences for optin methods of consent authorization. Fifth, confidentiality; how to assure respondents of confidentiality in an environment where there is significant suspicion of opaque third-party data access.

\section{Discussion}

Against the background of proliferating mHealth research and public health surveillance activities, which are 
sometimes difficult to distinguish, and that aim to reach larger numbers of individuals without in-person contact, we aimed to identify information that could be used to optimize consent approaches for future MPS. The main themes that were articulated through the interviews included potential ethical challenges and opportunities to conduct MPS for NCDs; consent processes and requirements for MPS-based data collection for public health surveillance versus research; the process and content of consent, consent authorization and issues of data privacy and confidentiality.

Respondents in this study raised concerns about the short duration of the IVR calls and the inability to assess comprehension of informed consent in MPS. For consent to be truly informed, it is imperative that participants understand the information disclosed to them before making a voluntary decision for or against participation. Cases have been documented where MPS protocols have been rejected by RECs because of the perception that there will not be sufficient opportunity for participants to comprehend survey disclosure information (22). With a remote investigator, it may be practically difficult to assess comprehension and voluntary choice (22). To mitigate these challenges, interviewees suggested that potential MPS respondents should be contacted prior to the actual survey either by text messaging or voice call to provide them with relevant information about the survey and give them time to understand. They also suggested that respondents should be given an option of calling back in case they decide to participate in the survey or providing a toll-free line, which they can call when they need any additional information.

Some KIs expressed the view that consent processes for MPS (in general, for some; and in particular when conducted as research, for others) should follow what is stipulated in the Uganda national ethics guidelines (23) regarding full disclosure, while others suggested that this disclosure information could be summarized, as it can be difficult for individuals to spend long periods of time on the phone. There is thus a tension, that is perhaps more acute than in "traditional" face-to-face surveys, between the amount of information to be provided during consent and the need to keep the survey short in MPS. Several KIs preferred use of "opt-in" forms of authorization (over opt-out) because they believed the population generally favored following positive instructions. Both opt-out and implied forms of authorization were regarded as confusing. Although not explicitly stated, several participants seemed sensitive to the need to be attentive to growing awareness amongst populations about a "right" to informed consent in health research, and were concerned about approaches to consent that might result in individuals perceiving a "violation" of this right. This observation reinforces the view that there is a growing awareness in Africa of the need to protect such rights (24).

While the majority of KIs interviewed believed in fully disclosing all basic elements of informed consent (25), there was consensus that disclosure language for MPS conducted exclusively for public health surveillance purposes need not to be as detailed as required for health research. To avoid inconveniencing respondents, KIs argued that consent procedures for MPS should not only include the necessary relevant information necessary for informed decision making, but should also be short and concise. These two desirable characteristics of consent would appear to be in conflict. Participant understanding during the consent process was not thought to be of paramount importance for NCD risk-factor surveillance, since the emphasis is usually on determinants of disease in the wider population. This seems to align with literature and international guidelines that suggest ethics norms and requirements of public health surveillance should and do differ from ethics norms and requirements of health research in general; though, as noted elsewhere, MPS may contribute to a blurring of the line between the two (26-28). Although much additional research on this subject is still needed, this study suggests that researchers and public health survey implementers contemplating using MPS may need to find a balance between providing enough information thus respecting the respondents right to an informed consent, without unduly burdening the respondents with a lengthy survey

Among the other ethical considerations that emerged were the issue of data security and the protection of the privacy and confidentiality of MPS respondents (29). The overall goal of effective data security is to protect individual identity of respondents and secure data in such a way that any inappropriate access or disclosure would not link the data with a particular person or with other data being sent (30). In some LMICs, the personal data collected is vulnerable to misuse due to poor or a lack of policies (31). These are pertinent societal issues which require broader discussions to allay the fears of the populace, increase understanding, and cultivate trust, if MPS programs are to gain wide acceptance for the benefit of all (12). It may be difficult to assure respondents of absolute confidentiality in MPS. Privacy is considered an essential freedom in Uganda. Individuals have the right to determine for themselves the when, how and extent of personal information to be 
communicated with others $(31,32)$. An individual can regulate access of such personal information through consent. Informed consent gives participants appropriate knowledge of the data to be collected, how it is stored and used, what rights they have to the data and the consequences or risks of disclosure (30). Unfortunately, relatively low technological literacy in Uganda limits people's discernment of the true risks and benefits of mobile technology (30). Mobile health data collection may increase the risk of social marginalization, psychological stress, invasion of privacy, or breach of confidentiality (33). Ethics committees and regulators therefore ought to develop systems that enhance the respondents' right to privacy while allowing room to collect population level information that may benefit the public good.

To our knowledge, this study is the first of its kind in sub-Saharan Africa with in-depth input from a range of stakeholders associated with MPS, in one country. The study highlights several key issues pertaining to consent for MPS that could potentially improve the ethical and regulatory quality of phone surveys aiming to identify NCD (and potentially other) risk factors within a population. The results of this exploratory study may not be representative of the wider Ugandan population because of participation of only purposively selected KIs; however, we hope the findings will help stimulate discussions and further research on the topic.

\section{Conclusions}

The increasing use of mobile devices and associated technologies globally offers a unique opportunity to transform data collection for monitoring disease burdens and risk factors at the population-level. However, there are concerns about appropriate consent options for mobile phone-based surveys conducted in LMICs. Voluntariness of decision making for participation in MPS is questionable because respondents may not have sufficient time to understand and ask questions. The consent language and authorization should be detailed enough to avail potential participants with adequate relevant information, but also not so lengthy as to discourage participation.

Ideally, participants should understand the information disclosed to them before making the decision to participate, but there is insufficient evidence of effective ways to assess comprehension though pre-recorded IVR MPS. Automated knowledge quizzes could be integrated, but without detailed explanation, they may further confuse respondents; and due to the increased burden of such quizzes and accompanying explanations, they may cause many types of MPS to become impracticable due to very low completion rates. Additional research is needed to address this challenge. "Opt-in" forms of authorization were considered superior to "optout" in Uganda because it was believed that the population, in general, prefers following positive instructions. Government authorities, policy makers, researchers, healthcare professionals and other stakeholders may utilize information from this study in the design of contextualized consent approaches for MPS. Although this research was conducted in Uganda, the general findings may be valid for neighboring countries, if not other LMIC environments with similar characteristics.

\section{Acknowledgments}

We would like to thank key informants and members of communities in Uganda for sharing their insight into this topic. We also thank Ms. Hannah Selig for her support with coordinating early activities related to this work.

Funding: This study was financially supported by the Bloomberg Philanthropies under the Data for Health Initiative (grant No. 119668). The funding agency had no role in the preparation of this manuscript.

\section{Footnote}

Conflicts of Interest: The authors have no conflicts of interest to declare.

Ethical Statement: The authors are accountable for all aspects of the work in ensuring that questions related to the accuracy or integrity of any part of the work are appropriately investigated and resolved. The study was approved by the Makerere University School of Public Health Higher Degrees and Research Ethics Committee (Protocol version 445/10/2017) and the Johns Hopkins Bloomberg School of Public Health Institutional Review Board; and was conducted in accordance with the provisions in the Helsinki Declaration. Informed consent was obtained from all participants prior to interview.

\section{References}

1. Pariyo GW, Wosu AC, Gibson DG, et al. Moving the Agenda on Noncommunicable Diseases: Policy Implications of Mobile Phone Surveys in Low and Middle- 
Income Countries. J Med Internet Res 2017;19:e115.

2. WHO. 2013 Global action plan for the prevention and control of noncommunicable diseases 2013-2020. 2013 [12/07/2018]. Available online: http://apps.who.int/iris/bit stream/10665/94384/1/9789241506236_eng.pdf?ua=1

3. UN. 2015. [20170102]. Resolution Adopted by the General Assembly on 25 September 2015. Available online: http://www.un.org/ga/search/view_doc.asp?symbol=A/ RES/70/1\&Lang=Ewebcite

4. Kay M, Santos J, Takane M. mHealth: New horizons for health through mobile technologies. World Health Organization 2011;64:66-71.

5. Barr ML, van Ritten JJ, Steel DG, et al. Inclusion of mobile phone numbers into an ongoing population health survey in New South Wales, Australia: design, methods, call outcomes, costs and sample representativeness. BMC Med Res Methodol 2012;12:177.

6. Liu B, Brotherton JM, Shellard D, et al. Mobile phones are a viable option for surveying young Australian women: a comparison of two telephone survey methods. BMC Med Res Methodol 2011;11:159.

7. Gibson DG, Pereira A, Farrenkopf BA, et al. Mobile Phone Surveys for Collecting Population-Level Estimates in Low- and Middle-Income Countries: A Literature Review. J Med Internet Res 2017;19:e139.

8. Labrique A, Blynn E, Ahmed S, et al. Health Surveys Using Mobile Phones in Developing Countries: Automated Active Strata Monitoring and Other Statistical Considerations for Improving Precision and Reducing Biases. J Med Internet Res 2017;19:e121.

9. Lukwago L, Nanyunja M, Ndayimirije N, et al. The implementation of Integrated Disease Surveillance and Response in Uganda: a review of progress and challenges between 2001 and 2007. Health Policy Plan 2013;28:30-40.

10. Somda ZC, Perry HN, Messonnier NR, et al. Modeling the cost-effectiveness of the integrated disease surveillance and response (IDSR) system: meningitis in Burkina Faso. PloS One 2010;5:e13044.

11. Vicente P, Reis E, Santos M. Using Mobile Phones for Survey Research: A Comparison with Fixed Phones. International Journal of Market Research 2009;51:1-16.

12. Ali J, Labrique AB, Gionfriddo K, et al. Ethics Considerations in Global Mobile Phone-Based Surveys of Noncommunicable Diseases: A Conceptual Exploration. J Med Internet Res 2017;19:e110.

13. Wolf SM, Clayton EW, Lawrenz F. The Past, Present, and Future of Informed Consent in Research and
Translational Medicine. Los Angeles, CA: SAGE Publications Sage CA, 2018.

14. Simon CM, Schartz HA, Rosenthal GE, et al. Perspectives on Electronic Informed Consent From Patients Underrepresented in Research in the United States: A Focus Group Study. J Empir Res Hum Res Ethics 2018;13:338-48.

15. Grady C, Cummings SR, Rowbotham MC, et al. Informed Consent. N Engl J Med 2017;376:856-67.

16. Furberg RD, Ortiz AM, Moultrie RR, et al. A Digital Decision Support Tool to Enhance Decisional Capacity for Clinical Trial Consent: Design and Development. JMIR Res Protoc 2018;7:e10525.

17. Data for Health. Available online: https://http://www. bloomberg.org/program/public-health/data-health/

18. Ellis JA. Leveraging Mobile Phones for Monitoring Risks for Noncommunicable Diseases in the Future. J Med Internet Res 2017;19:e137.

19. Gibson DG, Pariyo GW, Wosu AC, et al. Evaluation of Mechanisms to Improve Performance of Mobile Phone Surveys in Low- and Middle-Income Countries: Research Protocol. JMIR Res Protoc 2017;6:e81.

20. Braun V, Clarke V. Using thematic analysis in psychology. Qual Res Psychol 2006;3:77-101.

21. World Medical Association. World Medical Association Declaration of Helsinki: ethical principles for medical research involving human subjects. JAMA 2013;310:2191-4.

22. Parker M. Ethical considerations related to mobile technology use in medical research. J Mob Technol Med 2012;1:50-2.

23. UNCST. Uganda National Council for Science and Technology: National Guidelines for Research Involving Humans as Research Participants. Kampala, Uganda, 2014.

24. Ramsay M, de Vries J, Soodyall H, et al. Ethical issues in genomic research on the African continent: experiences and challenges to ethics review committees. Hum Genomics 2014;8:15.

25. Beauchamp TL, Childress J. Principles of Biomedical Ethics. 7th edition. New York: Oxford University Press, 2013.

26. Petrini C. Ethics of clinical science in a public health emergency: reflections on the role of research ethics boards. Am J Bioeth 2013;13:27-9.

27. WHO. WHO guidelines on ethical issues in public health surveillance. 2017.

28. Ali J, DiStefano MJ, Coates McCall I, et al. Ethics of mobile phone surveys to monitor non-communicable 
disease risk factors in low- and middle-income countries: A global stakeholder survey. Glob Public Health 2019;14:1167-81.

29. Filkins BL, Kim JY, Roberts B, et al. Privacy and security in the era of digital health: what should translational researchers know and do about it? Am J Transl Res 2016;8:1560-80.

30. Arora S, Yttri J, Nilse W. Privacy and Security in Mobile Health (mHealth) Research. Alcohol Res 2014;36:143-51.

31. Latif S, Rana R, Qadir J, et al. Mobile health in the

doi: $10.21037 /$ mhealth.2019.07.05

Cite this article as: Mwaka E, Nakigudde J, Ali J, Ochieng J, Hallez K, Tweheyo R, Labrique A, Gibson DG, Rutebemberwa E, Pariyo G. Consent for mobile phone surveys of noncommunicable disease risk factors in low-resource settings: an exploratory qualitative study in Uganda. mHealth 2019;5:26. developing world: Review of literature and lessons from a case study. IEEE Access 2017;5:11540-56.

32. National Information and Communications Technology Policy for Uganda (2014) [23/02/2019]. Available online: https://ict.go.ug/wp-content/uploads/2018/11/ICT Policy_2014.pdf

33. Labrique AB, Kirk GD, Westergaard RP, et al. Ethical issues in mHealth research involving persons living with HIV/AIDS and substance abuse. AIDS Res Treat 2013;2013:189645. 


\section{Key informant interview guide}

These questions/topics are a guide for key informant in-depth interviews with:

1. National/district officials responsible for the oversight of health research and/or public health surveillance;

2. Local academics and IRB staff who specialize in ethics, and

3. Technical experts in mobile phone surveys.

This is a guide and not to be used as a questionnaire but rather to explore these issues in a conversational way. The semistructured interviews should identify policies, experiences, opinions and preferences related to mobile phone surveys (both interactive voice response/IVR and short message service/SMS) for NCDs in terms of consent processes, disclosure language and methods of authorization. Be sure to record key points arising as well as non-verbal communication.

\section{Mobile phone surveys \& ethics background}

1. What is the nature of your previous experience with ethics or oversight of mHealth and more specifically, mobile phone surveys?

a. (For those with previous experience) What have been some of the ethics-related challenges that you have encountered in this context?

b. (For those with previous experience) What have been some of your experiences with the consent process for mobile phone surveys?

c. (Probe further as helpful to ease into discussion.)

I'm now going to provide a hypothetical scenario involving a mobile phone survey, which is based on actual activities. This is meant to help contextualize our discussion that follows.

\section{(Present hypothetical Mobile Phone Survey)}

A national IVR mobile phone survey of non-communicable disease (NCD) risk factors is being planned with adults aged 18 years and older in a low-income country. In IVR surveys, respondents interact with a pre-programmed database that contains both pre-recorded questions and a series of pre-set answers which are linked to specific numeric responses on a touch-tone phone keypad (e.g., "Press 1 for Yes"). The primary purpose of this survey is to inform future national health policy. The survey sample will be nationally representative. Participants will be selected and contacted through a process of combining digits at random to generate phone numbers (random digit dialing). The survey will be delivered in "official" local languages. No identifying data will be collected as part of the survey, though the raw data set will include the phone numbers that were dialed. Survey development and implementation will be led by a national governmental health agency in the country, with technical assistance from a high-income country partner and resources from global and high-income country funding bodies. There is potential for the collected data to be shared with multilateral organizations (e.g., WHO, UN).

\section{General impressions about consent}

2. What do you think are some of the potential challenges of doing consent for a mobile phone survey?

3. Can you think of any ways in which mobile phone surveys may enhance consent practices?

4. How might consent requirements change (if at all) depending on whether the mobile phone survey is being conducted for research $v s$. for public health surveillance?

a. Are there/should there be different consent requirements?

b. If so, what differences?

c. In your mind, what is the difference between the two?

\section{Disclosure of information during consent}

5. What information about a mobile phone survey is ethically required to be disclosed to respondents? 
6. What do you think are the main potential risks of mobile phone surveys for NCD?

7. Typically informed consent includes information about the purpose, procedures, potential risks, potential benefits, alternatives, confidentiality, compensation, contact persons and voluntary nature of participation. Individuals who conduct mobile phone surveys often times want to keep them as brief as possible to keep response burdens low. Do you have thoughts on how consent can be achieved under these constraints?

a. What are the elements of consent or informed consent that must be included in low-risk mobile phone surveys?

b. What are the elements of consent or informed consent (if any) that might be omitted in low-risk mobile phone surveys?

\section{Modes of authorization}

8. What options are available and appropriate for obtaining agreement or authorization to collect data from respondents? For example:

a. Opt-in vs. opt out (ex: "Press 1 if you would like to participate" vs. "Press 3 if you would not like to participate")

b. Passive $v s$. active (ex. "Press 1 if you would like to participate" vs. "By completing the survey you agree to participate")

9. Do you think particular modes of authorization are better than others? Why/why not?

\section{Other consent issues}

10. Do consent requirements (disclosure and authorization) change depending on the mobile phone delivery method (e.g., IVR vs. SMS)?

a. What considerations are specific to consent for an IVR mobile phone survey?

b. What considerations are specific to consent for a SMS mobile phone survey?

11. Do you think mobile phone survey practitioners are sufficiently trained and equipped to be able to develop appropriate consent approaches for mobile phone surveys?

12. Are research ethics committees or institutional review boards in the country sufficiently trained and equipped to be able to provide adequate review of consent approaches for mobile phone surveys?

a. What would support capacity strengthening?

13. Is there anything else you would like to share about consent and mobile phone surveys?

Thank you very much for your time. We appreciate your input.

Available for non-commercial use and adaptation. Please cite the accompanying manuscript if used or adapted. 